\title{
TIPOLOGÍAS Y CARACTERIZACIÓN DE SISTEMAS DE CRÍA BOVINA DE LA PAMPA DEPRIMIDA
}

\section{TYPOLOGIES AND CHARACTERIZATION OF COW-CALF SYSTEM THE FLOODING PAMPAS}

\author{
Claudia Faverin ${ }^{1,2 *}$ y Claudio Machado ${ }^{3}$ \\ ${ }^{1}$ Instituto Nacional de Tecnología Agropecuaria (INTA), Balcarce, Ruta 226 km 73,5 (CC 276), 7620 \\ Balcarce, Argentina. \\ ${ }^{2}$ Facultad de Ciencias Exactas y Naturales (UNMDP), Funes 3350, 7600 Mar del Plata, Argentina \\ ${ }^{3}$ Centro de Investigación Veterinaria de Tandil, Facultad de Ciencias Veterinarias, UNCPBA, CONICET \\ y CICBA), Paraje Arroyo Seco s/n, Campus Universitario, Tandil, Argentina \\ *Autor para correspondencia: faverin.claudia@inta.gob.ar
}

\section{RESUMEN}

La identificación y caracterización de los sistemas ganaderos permite acotar la diversidad y contribuir al proceso de toma de decisiones. Con el objetivo de identificar las tipologías de sistemas de cría bovina de la Pampa Deprimida se procesó la información de una encuesta de $\mathbf{1 1 5}$ predios utilizando 39 variables diagnósticas. Se analizaron datos de estructura de los establecimientos, indicadores productivos, recursos forrajeros y prácticas de alimentación animal, reproducción, prácticas sanitarias e información social. A partir de un análisis de conglomerados se establecieron cuatro grupos: G1: Productor tecnificado de escala media; G2: Productor tradicional de escala media; G3: Empresa tecnificada local de escala grande; y G4: Empresa tecnificada de escala grande. La carga animal media fue 0,72 vaca equivalente ha $^{-1}$. Los sistemas tecnificados presentaron mejores indicadores productivos que el tradicional. El principal recurso forrajero fue el campo natural, con mayor proporción en tradicionales $(98 \%)$ que en tecnificados $(67-72 \%)$. En éstos últimos la dieta también incluyó pasturas cultivadas $(9-19 \%)$, verdeos de invierno $(8,2-8,9 \%)$ y de verano $(4,7-5,5 \%)$. Los tecnificados difirieron principalmente en escala $(P<0,05)(499$ a 1627 ha), persona jurídica del propietario y la cercanía del lugar de residencia del titular y familia (urbana cercana o lejana según los grupos) entre otros, el reclutamiento de personal contratado temporariamente (75 a > 92\%), el uso y frecuencia de consultoría profesional, y prácticas aplicadas de salud animal, entre otros. Las tipologías obtenidas pueden contribuir al diseño de propuestas específicas de transferencia tecnológica por instituciones del sector.

Palabras clave: bovinos para carne, productor, sistemas pastoriles, análisis de conglomerados.

\section{ABSTRACT}

Identification and characterization of livestock systems help improve decision-making. In order to identify the typology of cow-calf systems from the Flooding Pampas, survey data of 115 farms were processed using 39 diagnostic variables. Data on farm structure, productivity indicators, forage resources, animal feeding practices, reproduction, animal health practices, and social information were analyzed. A cluster analysis was used to establish farm typologies. Four farm groups (G) were differentiated using cluster analysis: G1: Medium-scale technified farmer; G2: Mediumscale traditional farmer; G3: Large-scale technified local enterprise; and G4: Large-scale technified

Recibido: 04 junio 2018. Aceptado: 07 agosto 2018. 
enterprise. Overall mean stocking rate was 0.72 cow unit ha- ${ }^{-1}$. The technified systems had better productivity indicators than the traditional systems. The main fodder resource was grasslands, accounting for $98 \%$ and $67-72 \%$ of traditional $(98 \%)$ and technified $(67-72 \%)$ systems, respectively. The diet in technified systems also included cultivated pastures (9-19\%), and winter $(8.2-8.9 \%)$ and summer fodder crops $(4.7-5.5 \%)$. The technified systems mainly differed in terms of scale $(\mathrm{P}<0.05)$ (499 to $1627 \mathrm{ha}$ ), legal ownership, farmer's or farmer's family's place of residence (farm distance depending on G), recruitment of temporary staff $(75$ to $>92 \%)$, use and frequency of agricultural consultancy, adoption of different animal management practices, among others. This livestock farm typology may help agricultural institutions design specific technology transfer proposals.

Key words: Beef cattle, farmer, grazing systems, cluster analysis.

\section{INTRODUCCIÓN}

La Pampa Deprimida tiene gran relevancia en la cadena de producción de carne en Argentina; representa aproximadamente 9 millones de hectáreas en la provincia de Buenos Aires (Cid et al., 2011). Los sistemas de cría pastoriles son tradicionales en la región, donde el stock de terneros alcanza los 2,7 millones de cabezas, lo que representa el $48 \%$ del stock de la provincia de Buenos Aires (MinAgro, 2015). La Pampa Deprimida incluye las áreas conocidas como Cuenca del Salado (CS) y Depresión de Laprida (DL), que representan aproximadamente el 75 y el $25 \%$ de la región total, respectivamente.

Los sistemas de producción ganadera de CS y DL han sido caracterizados globalmente con datos del censo 2002 (Vázquez et al., 2008) y con paneles de expertos (Mosciaro et al., 2011). Del mismo modo, en los informes gubernamentales se definen establecimientos de cría promedio para estimar sus márgenes brutos (Arzubi et al., 2015) o emisiones de gases de efecto invernadero (TCN, 2015). A pesar que desde hace mucho tiempo se realizan importantes esfuerzos institucionales en la zona para una mayor transferencia tecnológica, en la región se observa una brecha enorme entre el conocimiento, la adopción de tecnología y las oportunidades de mercado (Arelovich et al., 2011; Pacin y Oesterheld, 2015; TCN, 2015). En este contexto, una descripción más precisa de las similitudes y diferencias de los sistemas ganaderos zonales puede contribuir al diseño y promoción de alternativas para lograr más eficiencia en el sector.

En la caracterización de un "establecimiento ganadero promedio", es importante considerar no sólo las condiciones productivas, sino también las sociales, culturales, económicas y/o ambientales (Solano et al., 2000; Milán et al., 2011). En ese sentido, el análisis tipológico de los productores es una herramienta de simplificación más integral que cuando se establecen sistemas modales basados solamente en análisis económicos (Alemu et al., 2016). Por lo tanto, este trabajo se orienta a clasificar los sistemas de cría de la Pampa
Deprimida con base en variables productivas y socio-económicas disponibles y de este modo generar información para facilitar el proceso de transferencia tecnológica en la región.

\section{MATERIALES Y MÉTODOS}

El área de estudio está ubicada en el CentroOeste de la Provincia de Buenos Aires, Argentina, en el área de influencia de las Estaciones Experimentales Agropecuarias (EEA) del INTA, Cuenca del Salado y un área de la EEA INTA Balcarce llamado Territorio Centro Oeste. En este trabajo la CS totaliza 6,5 millones de hectáreas, incluyendo los partidos (comunas) de Ayacucho, Maipú, General Madariaga, Tordillo, Castelli, General Guido, Mar Chiquita, General Lavalle, Dolores, Pinamar, Villa Gesell, Partido de la Costa, Pila, General Belgrano, Chascomús, Lezama, Punta Indio, Magdalena, Azul, Rauch, General Alvear, Tapalqué, Saladillo y Las Flores de la Provincia de Buenos Aires. Para el caso de la DL con 2,12 millones de hectáreas, se incluyen los partidos de Benito Juárez, Laprida, Olavarría, y Gral. Lamadrid de la Provincia de Buenos Aires.

La actividad ganadera bovina es la principal producción agropecuaria en ambas áreas, con predominancia de las razas Angus, Hereford y sus cruzas. El clima es templado y húmedo, con veranos e inviernos bien definidos, y heladas en invierno y primavera, con un período libre de heladas de 200-260 días (Otondo, 2011). La temperatura promedio anual varía entre $14-16^{\circ} \mathrm{C}$ y las precipitaciones disminuyen al sur-oeste (1.000-700 mm anuales). No hay una estación seca definida, pero las sequías son frecuentes en verano (altas temperaturas y vientos fuertes). La Pampa Deprimida posee una topografía plana, problemas de escurrimiento y de posibles encharcamientos.

La información utilizada corresponde a la Red de Información Agropecuaria Nacional (RIAN) del INTA y al período 1/7/2009 al 30/6/2010. La muestra corresponde a cuatro estratos de establecimientos definidos por el número de cabezas registradas en el Registro Nacional Sanitario de Productores 
Agropecuarios (RENSPA) (70-399, 400-1499, 15002999 y 3000 o más cabezas). La base de datos tenía 276 encuestas (221 para CS y 64 para la DL), realizadas a individuos, empresas y cooperativas de productores. Se trabajó con 115 (84 para CS y 31 para DL) debido a que este trabajo se restringió a productores con un solo establecimiento.

El cuestionario original incluyó información cualitativa y cuantitativa perteneciente a los tópicos: 1) Régimen de tenencia de la tierra, uso y superficie; 2) Caracterización del sistema productivo y de su manejo; 3) Alimentación del sistema ganadero; 4) Aspectos sanitarios; 5) Productos ganaderos; 6) Mejoras ganaderas; 7) Aspectos socio-económicos; 8) Asesoramiento técnico; 9) Capacitación de la empresa; 10) Aspectos que afectaron a la empresa en los últimos tres años.

En las Tablas 1 a 5 se presentan todas las variables seleccionadas y analizadas. Se realizó un análisis descriptivo de las variables por zona y para el área total (sólo se presentan medias y errores estándar por limitaciones de espacio). Las diferencias entre zonas fueron evaluadas mediante el test de $\mathrm{T}$ para muestras independientes para las variables continuas usando el PROC TTEST (SAS, 2009); y para las variables cualitativas se usó el test de ChiCuadrado (se usó el Test Exacto de Fisher cuando las frecuencias de las celdas fueron menores de 5) mediante el PROC FREQ (SAS, 2009). Después de estimar las correlaciones entre variables continuas, se realizó un análisis de agrupamiento jerárquico para separar los establecimientos, donde la matriz de distancia se construyó usando la métrica de Gower (Gower, 1971) con valores entre 0 (semejanza máxima) y 1 (semejanza mínima), que permite el uso combinado de variables cuantitativas y cualitativas. Los dendrogramas se infirieron usando el método de la Mínima Varianza de Ward (Ward, 1963) (no se presentan por limitaciones de espacio). La selección de variables finales se realizó en dos etapas: 1) mediante la selección de las variables significativas en cada uno de los seis conjuntos (Tablas 1 a 5), y 2) la selección de las variables representativas y significativas relacionadas a los conglomerados. Se aplicaron tests de Chi-Cuadrado para las tablas de contingencia entre conglomerados y cada variable cualitativa para cada etapa. Los procedimientos utilizados fueron el PROC DISTANCE para calcular la matriz de distancia, y PROC CLUSTER y PROC TREE para realizar el conglomerado y el dendrograma (SAS, 2009). El número de conglomerados seleccionados se determinó por apreciación visual y diferentes criterios: RMSSTD (desviación estándar agrupada de todas las variables que forman el grupo), SPRSQ (cuadrado $\mathrm{R}$ semi-parcial) y RSQ (R cuadrado). Después de obtener los conglomerados, se aplicó un modelo mixto usando el PROC MIXED (SAS, 2009) para el análisis de variables cuantitativas. El modelo incluyó el efecto del número del conglomerado $\mathrm{y}$, cuando fue necesario, se modeló la varianza de los mismos para solucionar problemas de heterogeneidad. La comparación de la proporción para las variables cualitativas se realizó mediante el Test de Chi-Cuadrado utilizando PROC FREQ (SAS, 2009) y se aplicó la corrección de Fisher cuando las frecuencias en las celdas fueron inferiores a 5 .

\section{RESULTADOS}

\section{Caracterización de los establecimientos por zona}

En las Tablas 1 a 5 se presentan los valores promedios y/o porcentajes para las variables evaluadas independiente de la zona de estudio. En líneas generales, pocas variables fueron diferentes entre zonas $(\mathrm{P}<0,05)$. El porcentaje de destete fue diferente entre CS y DL $(\mathrm{P}<0,05)$; el destete normal (5 a 7 meses de edad de los terneros) fue una práctica comúnmente adoptada en ambas zonas, aunque con una mayor adopción en DL (83,3\% de los casos) comparado con CS (55,4\% de los casos). Los tipos de destete temprano y anticipado (menos de 5 meses) fueron preferentemente adoptados en DL (9,7\% de los casos); en tanto que el destete tardío lo fue en CS (39,8\% de los casos). La alimentación animal se basó principalmente en pastizales naturales, con mayor representación en CS (78,4 \pm $2,8 \%$ de la superficie de los establecimientos) con respecto a DL $(64,3 \pm 4,7 \%)(\mathrm{P}<0,05)$, mientras que DL tuvo una mayor contribución de los verdeos de verano $(8,3 \pm 1,3 \%)$ que CS $(2,7 \pm 0,8 \%)(\mathrm{P}<0,05)$.

Las pasturas cultivadas fueron el segundo recurso alimenticio en importancia $(11,7 \pm 2,3 \%$ del área de los establecimientos para CS y 18,3 $\pm 3,7 \%$ para DL) y la superficie destinada a los verdeos de invierno fue similar entre las zonas (Tabla 2), siendo festuca (Festuca arundinacea Schreb), agropiro (Thinopyrum ponticum) y lotera (Lotus tenuis) las más comúnmente utilizadas. Los verdeos de invierno y verano más comunes fueron avena (Avena sativa) y raigrás (Lolium multiflorum), y sorgo (Sorghum bicolor), maíz (Zea mays) y moha (Setaria italica). La siembra convencional, directa o ambos tipos en DL se aplicaron a pasturas o cultivos en un 32,3\%, 38,7\% y $16,1 \%$ de los establecimientos, respectivamente. En la CS la siembra directa, convencional y ambos tipos se aplicó en el 39,3\%, 13,1\% y 13,1\% de los establecimientos, respectivamente. El 34,5\% de los encuestados indicaron que no realizaban ningún tipo de siembra en CS $(\mathrm{P}<0,05)$.

Entre las prácticas sanitarias usualmente aplicadas, solo el control de deficiencias minerales fue diferente entre zonas, donde en el 58,3\% de 


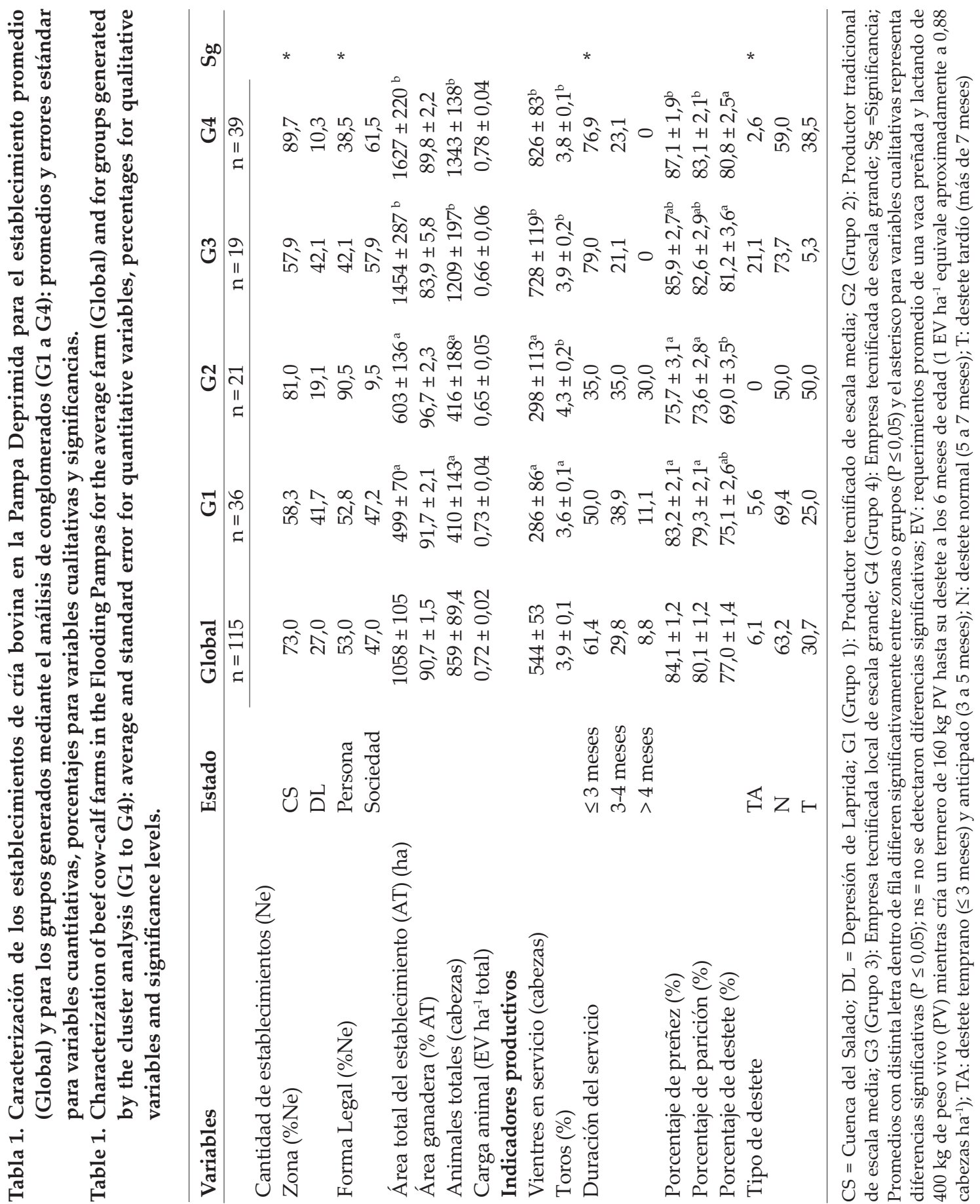

los establecimientos se realizaron están prácticas en la CS y sólo el 32,3\% lo hizo en la DL ( $\mathrm{P}<$ $0,05)$. Otra variable que resultó diferente entre zonas fue el lugar de residencia del productor; los productores viven en localidades cercanas a sus establecimientos $(67,7 \%$ para DL y $41,7 \%$ para CS) o en localidades lejanas en el caso de CS (40,5\%). El porcentaje de productores que viven en el campo es inferior al $18 \%$, siendo más frecuente en CS $(17,9 \%)$ que en DL $(12,9 \%)(\mathrm{P}<0,05)$. La ganadería, independientemente de la zona, constituye la mayor fuente de ingresos de estos productores (Tabla 4).

\section{Tipología de los establecimientos}

El análisis de conglomerados permitió 


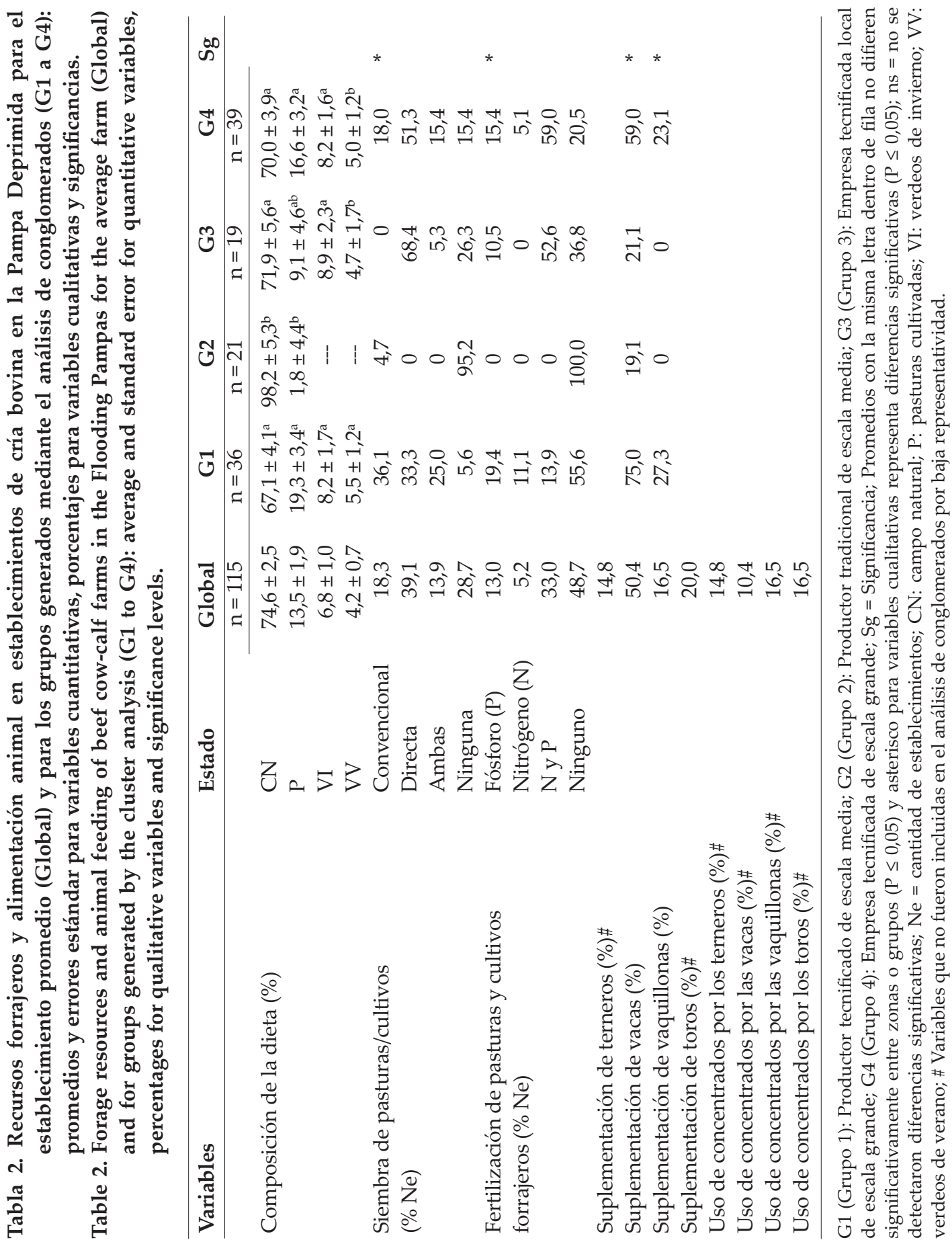


identificar cuatro grupos, un sistema tradicional y tres sistemas tecnificados diferenciados por la forma jurídica, escala y lugar de residencia del titular. En las Tablas 1 a 5 se presentan los valores promedios para todas las variables productivas y socioeconómicas por grupo. A continuación se mencionan las características principales de cada grupo.

El Grupo 1 (G1: Productor tecnificado de escala media) estuvo integrado por el $31 \%$ de la muestra e incluyó los productores con establecimientos de menor superficie (junto con el Grupo 2, Tabla 1) de CS y de DL. En el 89\% de los casos la duración del servicio fue inferior a 4 meses, con un porcentaje de preñez del $83 \%$ y con porcentajes de parición y destete de 79 y $75 \%$, respectivamente (Tabla 1). Aproximadamente el $70 \%$ de los establecimientos destetan cuando el ternero tiene entre 5 y 7 meses de edad y un $25 \%$ a mayores edades. La base forrajera incluye campo natural, pasturas cultivadas, verdeos de invierno y el mayor porcentaje de verdeos de verano con respecto a los otros grupos (Tabla 2). A proximadamente el 94,4\% de los productores siembran pasturas/cultivos en forma convencional y/o directa, y la casi mitad de ellos aplican fertilizantes $\mathrm{N}$ y/o P (Tabla 2). Este grupo presentó el mayor nivel de suplementación (heno o ensilaje) a vacas y vaquillonas (75 y $27 \%$, respectivamente) (Tabla 2). Los controles de enfermedades infecciosas, deficiencias minerales y el uso de antiparasitarios internos fueron prácticas de sanidad animal aplicadas con frecuencia moderada (Tabla 3). El productor y su familia reside en lugares urbanos cercanos, y la mano de obra (principalmente de tipo no familiar) es contratada temporalmente (Tabla 4). $\mathrm{El}$ asesoramiento veterinario fue cercano al $80 \%$, aunque casi el $50 \%$ de los productores realiza la contratación de forma ocasional (Tabla 5). El $50 \%$ de los productores no tiene asesoramiento agronómico, o si lo tiene es ocasional (Tabla 5).

El Grupo 2 (G2: Productores tradicionales de escala media) se localizó principalmente en la CS, y los propietarios eran básicamente personas físicas (Tabla 1). Este grupo incluye también los establecimientos de menor escala (junto con el grupo 1) con una media de 600 ha (Tabla 1). La duración del servicio fue la más extendida, pero el $70 \%$ de los productores aplicó un período inferior a 4 meses (Tabla 1). Los porcentajes de preñez y

Tabla 3. Mortalidad y prácticas sanitarias en establecimientos de cría bovina en la Pampa Deprimida para el establecimiento promedio (Global) y para los grupos generados mediante el análisis de conglomerados (G1 a G4): porcentajes para variables cualitativas y significancias.

Table 3. Mortality and sanitary practices of beef cow-calf farms in the Flooding Pampas for the average farm (Global) and for groups generated by the cluster analysis (G1 to G4): percentages for qualitative variables and significance levels.

\begin{tabular}{lcrrrrrr}
\hline Variables & Estado & Global & G1 & G2 & G3 & G4 & Sg \\
\hline & & $\mathrm{n}=115$ & $\mathrm{n}=36$ & $\mathrm{n}=21$ & $\mathrm{n}=19$ & $\mathrm{n}=39$ & \\
\cline { 2 - 6 } & & $3,9 \pm 0,7$ & & & & & \\
Mortalidad de vacas y toros (\%)\# & & $5,8 \pm 1,1$ & & & & & \\
Mortalidad de terneros (\%)\# & 1 & 8,7 & 11,1 & 28,6 & 0 & 0 & $*$ \\
Vacunación para enfermedades & 2 & 24,3 & 30,6 & 33,3 & 5,3 & 23,1 & \\
infecciosas (\%) & 3 & 33,9 & 38,5 & 15,4 & 2,6 & 43,6 & \\
& 4 & 33,0 & 16,7 & 9,5 & 89,5 & 33,3 & \\
Control de enfermedades & 0 & 19,1 & 36,1 & 23,8 & 0 & 10,3 & $*$ \\
reproductivas (\%) & 1 & 18,3 & 16,7 & 38,1 & 5,3 & 15,4 & \\
& 2 & 29,6 & 41,7 & 23,8 & 15,8 & 28,2 & \\
Control de deficiencias minerales (\%) & 3 & 33,0 & 5,6 & 14,3 & 79,0 & 46,2 & \\
Control de parásitos internos (\%) & & 51,3 & 41,7 & 23,8 & 78,9 & 61,5 & $*$ \\
& & 97,4 & 94,4 & 100 & 100 & 97,4 & $\mathrm{~ns}$ \\
\hline
\end{tabular}

G1 (Grupo 1): Productor tecnificado de escala media; G2 (Grupo 2): Productor tradicional de escala media; G3 (Grupo 3): Empresa tecnificada local de escala grande; G4 (Grupo 4): Empresa tecnificada de escala grande; Sg = Significancia; Promedios con la misma letra dentro de fila no difieren significativamente entre zonas o grupos $(P \leq 0,05)$ y asterisco para variables cualitativas representa diferencias significativas $(\mathrm{P} \leq 0,05)$; $\mathrm{ns}=$ no se detectaron diferencias significativas; Vacunación para enfermedades infecciosas: sumatoria del número de prácticas usadas en los establecimientos para el control/prevención de enfermedades infecciosas (vacunaciones para mancha y gangrena, carbunclo, diarrea neonatal y queratoconjuntivitis) (valores de 1 a 4); Control de enfermedades reproductivas: sumatoria del número de prácticas usadas en los establecimientos para el control/prevención de enfermedades reproductivas (enfermedades reproductivas, control de enfermedades venéreas y serología de la brucelosis) (Valores entre 0 y 3 ); \# Variables que no fueron incluidas en el análisis de conglomerados por baja representatividad. 
Tabla 4. Factores socio-económicos en establecimientos de cría bovina en la Pampa Deprimida para el establecimiento promedio (Global) y para los grupos generados mediante el análisis de conglomerados (G1 a G4): porcentajes para variables cualitativas y significancias.

Table 4. Socio-economic factors of beef cow-calf farms in the Flooding Pampas for the average farm (Global) and for groups generated by the cluster analysis (G1 to G4): percentages for qualitative variables and significance levels.

\begin{tabular}{|c|c|c|c|c|c|c|c|}
\hline Variables & Estado & Global & G1 & G2 & G3 & G4 & $\mathrm{Sg}$ \\
\hline & & $\mathrm{n}=115$ & $\mathrm{n}=36$ & $\mathrm{n}=21$ & $\mathrm{n}=19$ & $\mathrm{n}=39$ & \\
\hline \multirow[t]{3}{*}{ Residencia del productor (\%) } & $\mathrm{C}$ & 16,5 & 11,1 & 38,1 & 0 & 18,0 & \multirow[t]{3}{*}{ * } \\
\hline & UC & 48,7 & 83,3 & 33,3 & 94,7 & 2,6 & \\
\hline & UL & 34,8 & 5,6 & 28,6 & 5,3 & 79,5 & \\
\hline \multirow{3}{*}{$\begin{array}{l}\text { Residencia de la familia del } \\
\text { productor }(\%)\end{array}$} & $\mathrm{C}$ & 14,8 & 11,1 & 23,8 & 5,3 & 18,0 & \multirow[t]{3}{*}{ * } \\
\hline & UC & 49,6 & 83,3 & 42,9 & 89,5 & 2,6 & \\
\hline & UL & 35,7 & 5,6 & 33,3 & 5,3 & 79,5 & \\
\hline \multirow[t]{4}{*}{ Mano de obra permanente (\%) } & $\mathrm{F}$ & 6,96 & 8,3 & 9,5 & 5,3 & 5,1 & \multirow[t]{4}{*}{ ns } \\
\hline & NoF & 24,4 & 22,2 & 14,3 & 31,6 & 28,2 & \\
\hline & Ambos & 5,2 & 8,3 & 9,5 & 0 & 2,6 & \\
\hline & No tiene & 63,5 & 61,1 & 66,7 & 63,2 & 64,1 & \\
\hline \multirow[t]{4}{*}{ Mano de obra temporaria (\%) } & $\mathrm{F}$ & 15,7 & 13,9 & 38,1 & 10,5 & 7,7 & \multirow[t]{4}{*}{ * } \\
\hline & NoF & 53,9 & 41,7 & 38,1 & 79,0 & 61,5 & \\
\hline & Ambos & 19,1 & 19,4 & 19,1 & 10,5 & 23,1 & \\
\hline & No tiene & 11,3 & 25,0 & 4,7 & 0 & 7,7 & \\
\hline \multicolumn{3}{|c|}{ Ganadería como principal fuente de ingresos (\%)\# } & 84,2 & & & & \\
\hline
\end{tabular}

G1 (Grupo 1): Productor tecnificado de escala media; G2 (Grupo 2): Productor tradicional de escala media; G3 (Grupo 3): Empresa tecnificada local de escala grande; G4 (Grupo 4): Empresa tecnificada de escala grande; $S g=$ Significancia; Promedios con la misma letra dentro de fila no difieren significativamente entre zonas o grupos $(\mathrm{P} \leq 0,05)$ y asterisco para variables cualitativas representa diferencias significativas $(\mathrm{P} \leq 0,05)$; $\mathrm{ns}=$ no se detectaron diferencias significativas; C: campo; UC: urbana cercana; UL: urbana lejana; F: familiar; NoF: no familiar; \# Variables que no fueron incluidas en el análisis de conglomerados por baja representatividad.

parición fueron del 76 y 74\%, respectivamente; y registraron los menores porcentajes de destete (69\%) (Tabla 1). Los terneros tienen al menos 5 meses al momento del destete. La mayor parte de la superficie era de pastizales naturales y una muy pequeña proporción de pasturas (Tabla 2). La siembra de pasturas/cultivos se aplica en menos del $5 \%$ de los casos, y no se fertiliza (Tabla 2). Una baja proporción de productores utiliza heno y/o ensilaje como suplementos de las vacas (Tabla 2). Se observó una baja adopción de las prácticas de sanidad animal (Tabla 3). La mayor proporción de productores y sus familias viven en la zona rural, y la mano de obra es contratada temporalmente (Tabla 4). El grupo presentó el nivel más bajo de asistencia veterinaria, agronómica y de capacitación del productor y sus empleados (Tabla 5).

El Grupo 3 (G3: Empresa tecnificada local de escala grande) incluyó a los establecimientos más grandes (junto con el grupo 4) de la CS y de la DL, con una media de 1.450 ha (Tabla 1). La duración del servicio fue usualmente de 3 meses o menos (Tabla 1). Los porcentajes de preñez, parición y destete fueron altos con valores del 86,83 y $81 \%$, respectivamente. El destete se ejecuta a los 5 y 7 meses de edad, aunque el $21 \%$ de los productores lo realizan temprano o anticipadamente (Tabla 1). La base forrajera estuvo constituida por campo natural, pasturas cultivadas y verdeos (similar a G1 y G4) pero con ligeras diferencias entre su proporción (Tabla 2). La siembra de pasturas/cultivos fue principalmente en directa y las fertilizaciones $\mathrm{N}$ y $\mathrm{P}$ se utilizaron en el $53 \%$ de los casos (Tabla 2). Este grupo aplicó el mayor control sanitario (Tabla 3). El lugar de residencia de los productores y sus familias fue principalmente en una ciudad cercana (por lo que se la denomina local, para diferenciarla del grupo 4), el reclutamiento de mano de obra fue principalmente temporal y de tipo no familiar (Tabla 4) y presentó asesoramiento veterinario más permanente y agronómico ocasionalmente (Tabla 5).

El Grupo 4 (G4: Empresa tecnificada de escala grande) estuvo presente principalmente en la CS e involucró a los establecimientos de mayor escala (junto con el grupo 3) con una media de 1.620 hectáreas (Tabla 1). La duración del servicio fue usualmente de 3 meses o menos de duración 
Tabla 5. Asesoramiento y capacitación en establecimientos de cría bovina en la Pampa Deprimida para el establecimiento promedio (Global) y para los grupos generados mediante el análisis de conglomerados (G1 a G4): porcentajes para variables cualitativas y significancias.

Table 5. Advise and training in beef cow-calf farms in the Flooding Pampas for the average farm (Global) and for groups generated by the cluster analysis (G1 to G4): percentages for qualitative variables and significance levels.

\begin{tabular}{llcccccc}
\hline Variables & Estado & Global & G1 & G2 & G3 & G4 & Sg \\
\hline \multirow{2}{*}{ Asesoramiento veterinario (\%) } & & $\mathrm{n}=115$ & $\mathrm{n}=36$ & $\mathrm{n}=21$ & $\mathrm{n}=19$ & $\mathrm{n}=39$ & \\
& Permanente & 29,6 & 19,4 & 9,5 & 63,2 & 33,3 & $*$ \\
& Regular & 19,1 & 13,9 & 14,3 & 15,8 & 28,2 & \\
& Ocasional & 38,3 & 47,2 & 47,6 & 21,1 & 33,3 & \\
& No tiene & 13,0 & 19,4 & 28,6 & 0 & 5,1 & \\
Asesoramiento agronómico (\%) & Permanente & 19,1 & 11,1 & 0 & 26,3 & 33,3 & $*$ \\
& Regular & 11,3 & 5,6 & 0 & 21,1 & 18,0 & \\
& Ocasional & 27,0 & 33,3 & 4,8 & 36,8 & 28,2 \\
& No tiene & 42,6 & 50,0 & 95,2 & 15,8 & 20,5 & \\
Capacitación del productor y empleados (\%) & 34,8 & 41,7 & 4,7 & 42,1 & 41,0 & $*$ \\
\hline
\end{tabular}

G1 (Grupo 1): Productor tecnificado de escala media; G2 (Grupo 2): Productor tradicional de escala media; G3 (Grupo 3): Empresa tecnificada local de escala grande; G4 (Grupo 4): Empresa tecnificada de escala grande; Sg = Significancia; Promedios con la misma letra dentro de fila no difieren significativamente entre zonas o grupos $(P \leq 0,05)$ y asterisco para variables cualitativas representa diferencias significativas $(\mathrm{P} \leq 0,05)$; $\mathrm{ns}=$ no se detectaron diferencias significativas.

(Tabla 1). Los porcentajes de preñez, parición y destete fueron los más altos de todos los grupos $(87,1 ; 83,1 ;$ y $80,8 \%$, respectivamente, Tabla 1$)$. El destete fue cuando los terneros tenían entre 5 y 7 meses de edad o más tarde (Tabla 1). La superficie estuvo dedicada a campo natural, pasturas cultivadas y verdeos en proporciones similares al Grupo 1 (Tabla 2). El método de siembra de pasturas/cultivos fue principalmente directa, y la fertilización con N y P (Tabla 2). En el 59\% de los establecimientos se suplementa a las vacas (Tabla 2). La aplicación de prácticas sanitarias fue intermedia (Tabla 3). El lugar de residencia de los productores y sus familias estuvo situado lejos de los establecimientos (Tabla 4). La mano de obra se basó en empleados temporales no familiares, el asesoramiento veterinario (con diferente frecuencia) fue cercano al $95 \%$, y con alto nivel de agronómica, aunque con diferente tiempo de dedicación (Tabla 5).

\section{DISCUSIÓN}

\section{Enfoque metodológico}

La variedad y rango de datos, tanto cuantitativos como cualitativos, proporcionados por la diversidad de variables permitieron construir una caracterización de los tipos de establecimientos de la Pampa Deprimida. A nivel internacional se han utilizado diferentes métodos para identificar tipos de sistemas agrícola-ganaderos (Solano et al., 2000; Milán et al., 2011; Alemu et al., 2016). Para la zona de estudio se dispone de caracterizaciones simples
(Mosciaro et al., 2011; Némoz et al., 2013) y basadas en métodos multivariados (Costas et al., 2004; Vázquez et al., 2008), pero que incluyen menor número de variables y diferentes propósitos que en este estudio.

La condición distintiva y original del presente trabajoesla aplicación del análisisde conglomerados (uno de los métodos más ampliamente utilizados a nivel internacional) asociado con la estimación de la distancia de Gower. Esta última ha sido utilizada en estudios de biodiversidad genética en agricultura (Chávez Esponda et al., 2010; de Fatima Machado et al., 2011) y en estudios ecológicos (Sautot et al., 2015), y permite analizar conjuntamente las variables cuantitativas y cualitativas conservando su métrica inicial (SAS, 2013). Por lo tanto, el método estadístico utilizado permitió identificar grupos de establecimientos similares, y representa una herramienta prometedora para este tipo de estudios, para otros sistemas de producción o regiones.

\section{Tipología de los establecimientos}

Los establecimientos de cría en este estudio no mostraron mayores diferencias entre las zonas de CS y DL (Tablas 1 a 5). Los límites geográficos entre las zonas son claros, pero a veces hay pequeñas diferencias entre las estadísticas gubernamentales e informes en relación a los partidos que incluyen (MAGyP, 2011; Arzubi et al., 2015; MinAgro, 2015; TCN, 2015). Las observaciones sobre las características productivas (carga animal, porcentajes de parición y destete, y de producción 
animal en peso vivo, etc., Tabla 1) confirman la información disponible de CS (Maresca et al., 2011, Némoz et al., 2013) y de DL (Recavarren, 2016) lo que constituye una base descriptiva de la cría bovina de la zona.

Uno de los objetivos de los sistemas de cría es aumentar los $\mathrm{kg}$ de terneros destetados por vaca, lo que se procura mediante la mejora del rendimiento reproductivo de las vacas $y / 0$ la tasa de supervivencia de los terneros y su crecimiento (Beauchemin et al., 2011). La falta de concepción y las pérdidas de preñez oscilaron en promedio entre 13 y $24 \%$, y de 2,1 y $4,0 \%$, respectivamente (Tabla 1). Las mermas entre parición y destete variaron en promedio entre 4,8 y $8,2 \%$ (Tabla 1 ), donde el porcentaje de mortalidad de terneros promedio informado por los productores varió entre 4,7 y 6,9\% (Tabla 3). Estos porcentajes y su variación coinciden con lo informado por Maresca et al. (2011) a partir del monitoreo de la eficiencia reproductiva de rodeos de la CS (2004-2009), que mencionan porcentajes de mortalidad perinatal hasta los 20 días de edad de 3,9\% en vacas multíparas y $9,6 \%$ para primíparas.

El campo natural es uno de los principales recursos alimenticios para el ganado en el $79 \%$ de la superficie de los establecimientos (Tabla 2), tal como se ha informado para la CS (Cid et al., 2011). Sin embargo, la diversificación de la base forrajera a través de la inclusión de pasturas o cultivos anuales (Tabla 2), resulta una estrategia clave para mantener la carga con un buen rendimiento reproductivo, y poder hacer frente a la variabilidad estacional de la producción y de la calidad de la pradera (Némoz et al., 2013). Esta diversificación en la alimentación se observa más claramente mediante el análisis de tipologías, donde el Grupo 2 (Productores tradicionales de escala media) dependen mayormente de pastizales naturales (98\%) mientras que en el Grupo 1 representó solamente el $67 \%$ (Tabla 2).

Los sistemas extensivos con baja adopción de tecnología son frecuentes para la región estudiada (Vázquez et al., 2008; Némoz et al., 2013; MinAgro, 2015; TCN, 2015). Un informe reciente categorizó los sistemas de cría de la región en tradicionales y mejorados (Arzubi et al., 2015), donde los primeros se basan en pastizales naturales, tienen $0,58 \mathrm{EV} \mathrm{ha}^{-1}, 72 \%$ de destete $\mathrm{y}$ una producción de 92,1 $\mathrm{kg} \mathrm{PV} \mathrm{ha-1.}^{-1}$ Esto resulta similar a los $87,5 \mathrm{~kg} \mathrm{PV}^{-1} \mathrm{~h}^{-1}$ que pueden calcularse de grupo tradicional (Grupo 2, Tabla 1) que posee una gran dependencia de pastizales naturales, pero que en este trabajo representan menos del $20 \%$ de la muestra (Tabla 1). En el caso de los establecimientos mejorados, Arzubi et al. (2015) observaron una carga animal de $0,91 \mathrm{EV} \mathrm{ha}^{-1}$, un porcentaje de destete de $80 \%$ y $156,9 \mathrm{~kg}^{\mathrm{PV}} \mathrm{ha}^{-1}$. A partir del análisis de conglomerados realizado, los grupos 1,3 y 4 ( $82 \%$ de la muestra), representaron a los establecimientos mejorados (aquellos en los que los productores presentaron mayor adopción de diversas tecnologías, capacitación y contratación de asesoramiento técnico que los productores tradicionales). La producción estimada varió entre 110-117 $\mathrm{kg} \mathrm{PV} \mathrm{ha}^{-1}$, inferiores a los 156,9 kg PV ha ${ }^{-1}$ descritos por Arzubi et al. (2015). Sin embargo, cuando la misma estimación se limitó al 15\% superior en producción de carne de los establecimientos del presente estudio, se obtuvo una producción similar (152 $\left.\mathrm{kg} \mathrm{PV} \mathrm{ha}^{-1}\right)$. Es importante destacar que Arzubi et al. (2015) no informaron sobre la proporción de ambos tipos de establecimientos, lo cual sería útil para diseñar políticas específicas de promoción y extensión considerando la importancia de la región.

La contratación de mano de obra (temporaria no familiar) se asocia a la escala de los establecimientos, solo en el caso de los tecnificados (Tabla 1). Estos hallazgos coinciden con Némoz et al. (2013), que mencionan que los productores de menor escala tratan de simplificar las actividades agropecuarias siendo más reacios a contratar mano de obra permanente y asesoramiento técnico. La consulta a profesionales se considera clave ya que tiene un impacto positivo en la productividad de los establecimientos (Pacin y Oesterheld, 2015) lo cual también se observa en los resultados obtenidos. En promedio, el 57\% de las establecimientos (Grupos 1, 3 y 4) contrataron veterinarios de forma permanente o regular, mientras que en los establecimientos tradicionales (Grupo 2) esta cifra se redujo al 23\% (Tabla 1).

Los resultados (Tabla 1) ponen en evidencia la oportunidad tecnológica de mejora integral de la eficiencia de producción en los sistemas (Ej porcentaje de destete). Varios autores mencionan que una gestión efectiva que reduzca las pérdidas tiene un efecto sinérgico entre la aplicación de tecnología, la disminución de costos y el aumento de la producción (Ramsey et al., 2005; Burns et al., 2010). En el caso de los sistemas de la Pampa Deprimida, alcanzar mejores tasas de destete implicará no solo una mejora del manejo nutricional, sino también ajustar los programas sanitarios, lo que permite alcanzar una producción de 120 a $200 \mathrm{~kg} \mathrm{PV} \mathrm{ha}^{-1}$ año ${ }^{-1}$ (Rearte y Pordomingo, 2014). Sin embargo, aunque la brecha tecnológica para alcanzar rendimientos superiores requieren tecnologías de costo muy bajo (Arelovich et al., 2011), es importante mencionar que la información sobre la adopción de tecnología está fragmentada o no existe para la región estudiada (Cittadini et al, 2001). En ese sentido, aunque no están claras las implicancias de las diferencias observadas, por ejemplo sobre el lugar de 
residencia de los titulares y las estrategias de contratación de mano de obra, algunos estudios mencionan que estos factores podrían afectar la adopción tecnológica (Solano et al., 2006; Hostiou y Dedieu, 2012). Este y otros puntos, estimulan el desarrollo de investigaciones complementarias que aporten información específica de la zona para facilitar el diseño de propuestas de mejoras para las tipologías identificadas.

\section{CONCLUSIONES}

Los sistemas de producción de cría bovina en CS y DL presentaron similitud, pero el análisis de tipologías identificó cuatro grupos de establecimientos de los sistemas de cría bovina en la Pampa Deprimida: un sistema tradicional (Grupo 2: Productor tradicional de escala media) y tres sistemas tecnificados (Grupo 1: Productor tecnificada de escala media; Grupo 3: Empresa tecnificada local de escala grande; y Grupo 4: Empresa tecnificada de escala grande), con presencia diferencial en CS y DL. La clasificación realizada facilita el estudio complementario de oportunidades de innovación tecnológica para la mejora de la eficiencia en los sistemas de cría bovina de la Pampa Deprimida, de modo de contribuir a disminuir la brecha tecnológica con aquellos productores de la región que registran valores productivos superiores.

\section{RECONOCIMIENTOS}

Este trabajo es parte de la tesis doctoral del primer autor. Los autores desean agradecer al INTA por el soporte financiero (Beca de Postgrado, BASUR-1272101, y PNNAT-1128023) y a la UNMDP (AGR 413/13).También queremos agradecer a los Dres. E. Spath, C. Rossanigo, J. Fernández y al CPN M. Fernández por colaborar en el acceso a los datos de las encuestas, a las Sras. B. Masiero, A. Cano y a C.S. V. Frade por su contribución al análisis de datos, y al Ing. P. Recavarren, y al Dr. E. Melani por sus comentarios y sugerencias.

\section{LITERATURA CITADA}

Alemu, A.K., B.D. Amiro, S. Bittman, D. MacDonald, and K.M. Ominiski. 2016. A typological characterization of Canadian beef cattle farms based on a producer survey. Can. J. Anim. Sci. 96:187-202. doi:10.1139/ cjas-2015-0060.

Arelovich, H.M., R.D. Bravo, and M.F. Martínez. 2011. Development, characteristics, and trends for beef cattle production in Argentina. Animal Frontiers 1(2):37-45. doi:10.2527/ af.2011-0021.
Arzubi, A., R. Vidal, y J. Moares. 2015. Boletín Trimestral Bovino $N^{\circ} 14.75$ p. Subsecretaría de Ganadería. Secretaría de Agricultura, Ganadería y Pesca. Ministerio de Agricultura, Ganadería y Pesca, Buenos Aires, Argentina.

Beauchemin, K.A., H.H. Janzen, S.M. Little, T.A. McAllister, and S.M. McGinn. 2011. Mitigation of greenhouse gas emissions from beef production in western Canada - Evaluation using farm-based life cycle assessment. Animal Feed Science and Technology 166-167:663- 677. doi:10.1016/j. anifeedsci.2011.04.047.

Burns, B.M., Fordyce, G., and R.G. Holroy. 2010. A review of factors that impact on the capacity of beef cattle females to conceive, maintain a pregnancy and wean a calf-Implications for reproductive efficiency in northern Australia. An. Reprod. Sci. 122:1-22. doi:10.1016/j. anireprosci.2010.04.010

Chávez Esponda, D., I. Miranda Cabrera, M. Varela Nualles, y L. Fernández. 2010. Utilización del análisis de cluster con variables mixtas en la selección de genotipos de maíz (Zea mays). Revista Investigación Operacional 31:209-216.

Cid, M.S., R.C. Fernández, M. Oesterheld, J.M. Paruelo, A.F. Cibils, and M.A. Brizuela. 2011. Grass-fed beef production systems of Argentina's flooding pampas. Understanding ecosystem heterogeneity to improve livestock production. Outlook Agr. 40(2):181-189. doi: 10.5367/oa.2011.0040.

Cittadini, R., J. Burges, V. Hamdan, P. Natinzon, R. Pérez, y B. Dedieu. 2001. Diversidad de sistemas ganaderos y su articulación con el sistema familiar. Rev. Arg. Prod. Anim. 21(2):119-135.

Costas, A.M., N.N. Abbiati, y A.M. Pereyra. 2004. Requerimientos y producción propia de alimentos de ganadería pastoril: una tipología en la Provincia de Buenos Aires. Arch. Latinoam. Prod. Anim. 12(1):28-4.

De Fatima Machado, C., F.V.D. Souza, J.R.S. Cabral; C.A. Da Silva Leo, A.P. De Matos, and R. Ritzinger. 2011. Cluster analysis using quantitative, qualitative and molecular traits for the study of the genetic diversity in pineapple genotypes. Acta Hortic 902:159-162.

Gower, J.C. 1971. A general coefficient of similarity and some of its properties. Biometrics 27:857874.

Hostiou, N., and B. Dedieu. 2012. A method for assessing work productivity and flexibility in livestock farms. Animal 6(5):852-862. doi:10.1017/S1751731111002084. 
MAGyP. 2011. Ganados y Carnes. Anuario 2010. Ministerio de Agricultura, Ganadería y Pesca (MAGyP). 480 p. Secretaría de Agricultura, Ganadería y Pesca, Subsecretaría de Ganadería, Buenos Aires, Argentina.

Maresca, S., J. Quiroz García, y F. Plorutti. 2011. Eficiencia reproductiva en rodeos de cría de la Cuenca del Salado. 55 p. Ediciones INTA, Centro Regional Buenos Aires Sur, Estación Experimental Agropecuaria Cuenca del Salado, Rauch, Argentina.

Milán, M.J., G. Caja, R. González-González, A.M. Fernández-Pérez, and X. Such. 2011. Structure and performance of Awassi and Assaf dairy sheep farms in northwestern Spain. J. Dairy Sci. 94:771-784. doi: 10.3168/jds.2010-3520.

MinAgro. 2015. Caracterización de la producción bovina. Buenos Aires - Corrientes - Chaco - Formosa - La Pampa- Misiones. 193 p. Sistema de Monitoreo del Sector de la Carne Bovina. Serie 1. Ministerio de Agroindustria (MinAgro), Presidencia de la Nación, Subsecretaria de Ganadería, Buenos Aires, Argentina.

Mosciaro, M., P. Natinzon, y J.C. Tosi. 2011. Análisis de la situación actual y de la sustentabilidad económica de sistemas característicos de los territorios del CERBAS. Informe de PR BASUR 720071 y PE AESS-302443. Disponible en www.inta.gob.ar/sites/default/files/scripttmp-modelos_ucts.pdf (Consulta 14 junio 2017).

Némoz, J.P., S.I. Giancola, M.S.Bruno, M.B. De La Vega, S.Calvo, S. Di Giano, et al. 2013. Causas que afectan la adopción de tecnología en la ganadería bovina para carne de la Cuenca del Salado: enfoque cualitativo. Estudios socioeconómicos de la adopción de tecnología № 5. Ediciones INTA, Buenos Aires, Argentina.

Otondo, J. 2011. Efectos de la introducción de especies megatérmicas sobre características agronómicas y edáficas de un ambiente halomórfico de la Pampa Inundable. Tesis de Magister Scientiae. Escuela para Graduados Alberto Soriano. Facultad de Agronomía, Universidad de Buenos Aires, Argentina.

Pacin, F., and M. Oesterheld. 2015. Closing the technological gap of animal and crop production through technical assistance. Agr. Syst. 137:101-107. doi: 10.1016/j. agsy.2015.04.007.

Ramsey, R., D. Doye, C. Ward, J. McGrann, L. Falconer, and S. Bevers. 2005. Factors affecting beef cow-herd costs, production, and profits. J. Agr. Appl. Econ. 37:91-99. doi: 10.1017/S1074070800007124
Rearte, D.H., and A.J. Pordomingo. 2014. The relevance of methane emissions from beef production and the challenges of the Argentinean beef production platform. Meat Science 98:355-360. doi:10.1016/j. meatsci.2014.06.021.

Recavarren, P. 2016. La producción agropecuaria en Olavarría, Benito Juárez, Laprida y Gral. La Madrid: evolución y desafíos a futuro. Ediciones INTA, Balcarce, Buenos Aires, Argentina. Disponible en http://inta.gob. ar/sites/default/files/inta_la_produccion_ agropecuaria_ en_olavarria_juarez_laprida_ la_madrid_2016.pdf (Consulta 14 Junio 2017).

SAS. 2009. Base SAS 9.2 Procedures Guide: Statistical Procedures. $2^{\text {nd }}$. ed. SAS, Cary, North Carolina, USA.

SAS. 2013. SAS Institute Inc. 2013.SAS/STAT® 13.1 User's Guide. SAS, Cary, North Caroline, USA.

Sautot, L., B. Faivre, L. Journaux, and P. Molin. 2015. The hierarchical agglomerative clustering with Gower index: A methodology for automatic design of OLAP cube in ecological data processing context. Ecol. Inform. 26:217230. doi: 10.1016/j.ecoinf.2014.07.011.

Solano, C., A. Bernués, F. Rojas, N. Joaquín, W. Fernández, and M. Herrero. 2000. Relationships between management intensity and structural and social variables in dairy and dual-purpose systems in Santa Cruz, Bolivia. Agr. Syst. 65:159-177.

Solano, C., H. León, E. Pérez, L. Tole, R.H. Fawcett, and M. Herrero. 2006. Using farmer decisionmaking profiles and managerial capacity as predictors of farm management and performance in Costa Rican dairy farms. Agr. Syst. 88:395- 428.

TCN. 2015. Inventario de Gases de Efecto Invernadero de la República Argentina. Año 2012. Agricultura, Ganadería y Cambio de Uso de la Tierra. Tercera Comunicación Nacional de la República Argentina a la Convención Marco de las Naciones Unidas sobre Cambio Climático. 264 p. Asociación Argentina de Consorcios Regionales de Experimentación Agrícola (AACREA), Fundación Torcuato Di Tella (FTDT), Price Waterhouse \& Co. Asesores de Empresas SRL (PwC), Buenos Aires, Argentina.

Vázquez, P., M. Rojas, y J.C. Burges. 2008. Caracterización y tendencias de la ganadería bovina en la Cuenca del Salado. Veterinaria Argentina 25(248):572-584.

Ward, J.H. 1963. Hierarchical grouping to optimize an objective function. J. Am. Stat. Assoc. 58:236-244. 